\title{
ON THE SOLVABILITY OF BOUNDARY VALUE PROBLEMS FOR SOME NONLINEAR SYSTEMS OF ORDINARY DIFFERENTIAL EQUATIONS ON $\mathbb{R}_{+}$
}

\author{
JITKA VACKOVÁ \\ Received 22 September, 2016
}

\begin{abstract}
Sufficient conditions guaranteeing the unique existence of a bounded solution of quasilinear boundary value problems are established and a successive approximation method for constructing the solution is described, together with the proof of the stability of the used method.
\end{abstract}

2010 Mathematics Subject Classification: 34B10; 34B15

Keywords: boundary value problems, existence, uniqueness and construction of solution, Carathéodory conditions, stability

\section{INTRODUCTION}

On $\mathbb{R}_{+}$we consider the boundary value problem for the quasi-linear differential system:

$$
\begin{aligned}
x^{\prime}(t) & =P(t) x(t)+f(t, x(t)), \\
\ell(x) & =h(x),
\end{aligned}
$$

where $P \in L_{\text {loc }}\left(\mathbb{R}_{+}, \mathbb{R}^{n \times n}\right), \quad \ell: C_{\text {loc }}\left(\mathbb{R}_{+}, \mathbb{R}^{n}\right) \rightarrow \mathbb{R}^{n}$ is linear bounded functional, $h: C_{\text {loc }}\left(\mathbb{R}_{+}, \mathbb{R}^{n}\right) \rightarrow \mathbb{R}^{n}$ is continuous and generally nonlinear operator and $f: \mathbb{R}_{+} \times$ $\mathbb{R}^{n} \rightarrow \mathbb{R}^{n}$ is a function satisfying the local Caratheodory conditions :

(1) $f(\cdot, x): \mathbb{R}_{+} \rightarrow \mathbb{R}^{n}$ is a measurable function for an arbitrary $x \in \mathbb{R}^{n}$,

(2) $f(t, \cdot): \mathbb{R}^{n} \rightarrow \mathbb{R}^{n}$ is a continuous function for almost every $t \in \mathbb{R}_{+}$,

(3) $\forall r>0 \exists k_{r} \in L\left(\mathbb{R}_{+} ; \mathbb{R}_{+}\right)$such that $\|f(t, x)\| \leq k_{r}(t)$ for almost every $t \in \mathbb{R}_{+}$ and $x \in \mathbb{R}^{n}$ such that $\|x\| \leq r$.

If we put $f(t, x(t)) \equiv q(t)$ and $h(x) \equiv c_{0}$ we get from the problem (1.1), (1.2) the linear boundary value problem for ordinary differential equations on $\mathbb{R}_{+}$which was studied in [3].

The solvability of boundary value problems for ordinary differential systems on $\mathbb{R}_{+}$is studied in much bibliography, for example in monograph [1] and publications $[3,5,7]$ and in references mentioned there. The construction methods of the solution 
of boundary value problems for systems of ordinary linear differential equations on closed interval is studied for example in §4.5. of monograph [4].

In Miskolc Mathematical Notes there are authors studying similar problems, the solutions of quasilinear differential equations on $\mathbb{R}_{+}$in publication [2] and problems of integral-differential equations in publication [8].

In this work Theorems 1 and 2 provide the criteria of solvability and uniqueness solvability including the construction method of successive aproximations for generally nonlinear boundary value problems on $\mathbb{R}_{+}$. Theorem 3 proves the stability of the method used for constructing the solution.

By a solution of system (1.1) on $\mathbb{R}_{+}$it is uderstood a local absolutely continuous vector function $x \in C_{\text {loc }}\left(\mathbb{R}_{+}, \mathbb{R}^{n}\right)$ which is bounded on $\mathbb{R}_{+}$, i.e.

$$
\sup \left\{\|x(t)\|: t \in \mathbb{R}_{+}\right\}<+\infty,
$$

and satisfies the equation (1.1) for almost every $t \in \mathbb{R}_{+}$. A solution of system (1.1) satisfying condition (1.2) is called a solution of problem (1.1), (1.2) on $\mathbb{R}_{+}$.

Basic notation:

$\mathbb{R}=(-\infty,+\infty), \mathbb{R}_{+}=[0, \infty)$,

$\mathbb{R}^{n}$ - the space of $\mathrm{n}$-dimensional column vectors $x=\left(x_{i}\right)_{i=1}^{n}$ with the norm

$$
\|x\|=\sum_{i=1}^{n}\left|x_{i}\right| \quad \text { and } \quad|x|=\left(\left|x_{i}\right|\right)_{i=1}^{n},
$$

$\mathbb{R}_{+}^{n}=\left\{x=\left(x_{i}\right)_{i=1}^{n} \in \mathbb{R}^{n}: x_{i} \in \mathbb{R}_{+}(i=1, \ldots, n)\right\}$,

$\mathbb{R}^{n \times n}$ - the space of $n \times n$ matrices $X=\left(x_{i j}\right)_{i, j=1}^{n}$ with the norm

$$
\|X\|=\sum_{i, j=1}^{n}\left|x_{i j}\right| \quad \text { and } \quad|X|=\left(\left|x_{i j}\right|\right)_{i, j=1}^{n},
$$

$\mathbb{R}_{+}^{n \times n}=\left\{X=\left(x_{i j}\right)_{i, j=1}^{n} \in \mathbb{R}^{n \times n}: x_{i j} \in \mathbb{R}_{+}(i, j=1, \ldots, n)\right\}$.

if $x, y \in \mathbb{R}^{n}$ and $X, Y \in \mathbb{R}^{n \times n}$, then

$$
x \leq y \Longleftrightarrow y-x \in \mathbb{R}_{+}^{n}, \quad X \leq Y \Longleftrightarrow Y-X \in \mathbb{R}_{+}^{n \times n} .
$$

For the matrix $X=\left(x_{i j}\right)_{i, j=1}^{n} \in \mathbb{R}^{n \times n}$ by the notation $X_{d}$ and $X_{\bar{d}}$ we mean

$$
X_{d}=\left(\begin{array}{cccc}
x_{1} & 0 & \ldots & 0 \\
0 & x_{2} & \ldots & 0 \\
\ldots \ldots & \ldots & \ldots \\
0 & 0 & \ldots & x_{n}
\end{array}\right), \quad X_{\bar{d}}=\left(\begin{array}{cccc}
0 & x_{12} & \ldots & x_{1 n} \\
x_{21} & 0 & \ldots & x_{2 n} \\
\ldots \ldots & \ldots \ldots \ldots . . . \\
x_{n 1} & x_{n 2} & \ldots & 0
\end{array}\right),
$$

$\operatorname{det}(X)$ - the determinant of the matrix $X$, $r(X)$ - the spectral radius of the matrix $X$, $X^{-1}$ - the inverse matrix to $X$,

$E$ - the unit matrix, 
$C\left(\mathbb{R}_{+}, \mathbb{R}^{n}\right)$ - the space of continuous and bounded vector functions ${ }^{1} x: \mathbb{R}_{+} \rightarrow \mathbb{R}^{n}$ with the norm

$$
\|x\|_{C}=\sup \left\{\|x(t)\|: t \in \mathbb{R}_{+}\right\} \quad \text { and } \quad|x|_{C}=\left(\left\|x_{i}\right\|_{C}\right)_{i=1}^{n},
$$

$L\left(\mathbb{R}_{+}, \mathbb{R}^{n}\right)$ - the space of integrable vector functions $x: \mathbb{R}_{+} \rightarrow \mathbb{R}^{n}$ with the norm

$$
\|x\|_{L}=\int_{0}^{+\infty}\|x(t)\| d t \quad \text { and } \quad|x|_{L}=\left(\left|x_{i}\right|_{L}\right)_{i=1}^{n},
$$

$L\left(\mathbb{R}_{+}, \mathbb{R}^{n \times n}\right)$ - the space of integrable matrix functions $X: \mathbb{R}_{+} \rightarrow \mathbb{R}^{n \times n}$ with the norm

$$
\|X\|_{L}=\int_{0}^{+\infty}\|X(t)\| d t \quad \text { and } \quad|X|_{L}=\left(\left|x_{i j}\right|_{L}\right)_{i, j=1}^{n},
$$

if $X \in C\left(\mathbb{R}_{+}, \mathbb{R}^{n \times n}\right)$ is a matrix function with columns $x_{1}, \ldots, x_{n}$ and $\ell: C\left(\mathbb{R}_{+}, \mathbb{R}^{n}\right) \rightarrow \mathbb{R}^{n}$ is a linear functional, then $\ell(X)$ stands for the matrix function with columns $\ell\left(x_{1}\right), \ldots, \ell\left(x_{n}\right)$.

\section{Results}

To formulate the Theorem on solvability of the problem (1.1), (1.2) we need to formulate the following estimates $\forall x, y \in C_{0}\left(\mathbb{R}_{+}, \mathbb{R}^{n}\right)$ :

$$
|f(t, x)-f(t, y)| \leq \bar{L}(t)|x-y| C
$$

where $\bar{L}(t) \in L\left(\mathbb{R}_{+}, \mathbb{R}^{n \times n}\right)$,

$$
|h(x)-h(y)| \leq H|x-y|_{C}
$$

where $H \in \mathbb{R}^{n \times n}$ is constant matrix and

$$
|\ell(x)| \leq \bar{\ell}(|x|),
$$

where $\bar{\ell}: C_{0}\left(\mathbb{R}_{+}, \mathbb{R}^{n}\right) \rightarrow \mathbb{R}^{n}$ is linear positive bounded functional. Moreover we use the notations:

$$
\begin{aligned}
& P_{d}^{*}=\sup \left\{\int_{t_{0}}^{t} P_{d}(s) d s: t \in \mathbb{R}_{+}\right\}, \\
& P^{*}=\sup \left\{\left|\int_{t_{0}}^{t} \exp \left(\int_{s}^{t} P_{d}(\xi) d \xi\right)\right| P_{\bar{d}}(s)|d s|: t \in \mathbb{R}_{+}\right\}, \\
& f^{*}=\sup \left\{\left|\int_{t_{0}}^{t} \exp \left(\int_{s}^{t} P_{d}(\xi) d \xi\right)\right| \bar{L}(s)|d s|: t \in \mathbb{R}_{+}\right\},
\end{aligned}
$$

where $t_{0} \in \mathbb{R}_{+}$.

\footnotetext{
${ }^{1} \mathrm{~A}$ vector or matrix function is said to be continuous, bounded, integrable etc., if such are its elements
} 
Theorem 1. Assume that $P_{d}^{*}, P^{*}$ and $\bar{\ell}(E)$, defined previously, satisfy the condition

$$
\left\|P_{d}^{*}\right\|+\left\|P^{*}\right\|+\|\bar{\ell}(E)\|<+\infty
$$

the matrix

$$
\Lambda=\ell\left(\exp \int_{t_{0}}^{\cdot} P_{d}(s) d s\right) \quad \text { is nonsingular }
$$

and

$$
r(S)<1
$$

where

and

$$
S=P^{*}+f^{*}+\Theta_{d}^{*}\left|\Lambda^{-1}\right|\left[H+\bar{\ell}(E)\left(P^{*}+f^{*}\right)\right],
$$

$$
\Theta_{d}^{*}=\sup \left\{\exp \int_{t_{0}}^{t} P_{d}(\xi) d \xi: t \in \mathbb{R}_{+}\right\} .
$$

Then the problem (1.1), (1.2) has just one solution.

To prove Theorem 1 we shall need to formulate the following statement that corresponds with the methods used in publications of I.Kiguradze (see $[4,5,7]$ and the bibliography mentioned there). To get the effective criteria of solvability of the considered problem we use one of the methods presented in $§ 1.3$. of [6].

Proposition 1. Every solution of the problem (1.1), (1.2) is also a solution of an operator equation

$$
x=T x
$$

where

$$
\begin{aligned}
T x= & \int_{t_{0}}^{t} \exp \left(\int_{s}^{t} P_{d}(\xi) d \xi\right)\left[P_{\bar{d}}(s) x(s)+f(s, x(s))\right] d s+\exp \left(\int_{t_{0}}^{t} P_{d}(\xi) d \xi\right) \\
& \times \Lambda^{-1}\left[h(x)-\ell\left(\int_{t_{0}} \exp \left(\int_{s} P_{d}(\xi) d \xi\right)\left[P_{\bar{d}}(s) x(s)+f(s, x(s))\right] d s\right)\right]
\end{aligned}
$$

where $t_{0} \in \mathbb{R}_{+}$is an arbitrary fixed point. And from the other side, every solution of the operator equation (2.5) is also a solution of the problem (1.1), (1.2).

Proof. Let $x(t)$ be a solution of the problem (1.1), (1.2). Then by integration of (1.1) we get

$$
\begin{aligned}
x(t)= & x\left(t_{0}\right) \exp \left(\int_{t_{0}}^{t} P_{d}(\xi) d \xi\right) \\
& +\int_{t_{0}}^{t} \exp \left(\int_{s}^{t} P_{d}(\xi) d \xi\right)\left[P_{\bar{d}}(s) x(s)+f(s, x(s))\right] d s .
\end{aligned}
$$


To calculate $x\left(t_{0}\right)$ we shall substitute $\mathrm{x}(\mathrm{t})$ in the left side of the boundary condition (1.2) and with use of the linearity of the functional $\ell$ we get

$$
\begin{aligned}
h(x)= & \ell\left(\exp \left(\int_{t_{0}}^{\cdot} P_{d}(\xi) d \xi\right)\right) x\left(t_{0}\right) \\
& +\ell\left(\int_{t_{0}}^{\cdot} \exp \left(\int_{s} P_{d}(\xi) d \xi\right)\left[P_{\bar{d}}(s) x(s)+f(s, x(s))\right] d s\right) .
\end{aligned}
$$

Then according to (2.2) we obtain

$$
x\left(t_{0}\right)=\Lambda^{-1}\left(h(x)-\ell\left(\int_{t_{0}}^{\cdot} \exp \left(\int_{s} P_{d}(\xi) d \xi\right)\left[P_{\bar{d}}(s) x(s)+f(s, x(s))\right] d s\right)\right) .
$$

By substituting $x\left(t_{0}\right)$ back in (2.7) we get (2.6).

From the other side, if $x(t)$ is a solution of (2.5) then it satisfies the condition (1.2), because by substituting $t_{0}$ for $t$ in (2.6) we get:

$$
x\left(t_{0}\right)=\Lambda^{-1}\left[h(x)-\ell\left(x(\cdot)-x\left(t_{0}\right) \exp \left(\int_{t_{0}}^{t} P_{d}(\xi) d \xi\right)\right)\right],
$$

and then with use of linearity of $\ell$ we get (1.2). By derivating of (2.5) we get equation (1.1). It means that the solution $x(t)$ is also the solution of the problem (1.1), (1.2).

Proof of Theorem 1. To deduce the sufficient criterias of the solvability of the equation (2.5) we have to prove the contractivity of the operator $T: C_{0}\left(\mathbb{R}_{+}, \mathbb{R}^{n}\right) \rightarrow$ $C_{0}\left(\mathbb{R}_{+}, \mathbb{R}^{n}\right)$, where $C_{0}\left(\mathbb{R}_{+}, \mathbb{R}^{n}\right)$ is a subspace of the space $C_{\text {loc }}\left(\mathbb{R}_{+}, \mathbb{R}^{n}\right)$ with bounded functions on $\mathbb{R}_{+}$. This space is a Banach space with a supreme norm and thus the operator $T$ displays a Banach space into itself. Let's make the following estimation:

$$
\begin{aligned}
|T x-T y|_{C} \leq & \left|\int_{t_{0}}^{t} \exp \left(\int_{s}^{t} P_{d}(\xi) d \xi\right) P_{\bar{d}}(s)[x(s)-y(s)]\right|_{C} \\
& +\left|\int_{t_{0}}^{t} \exp \left(\int_{s}^{t} P_{d}(\xi) d \xi\right)[f(s, x(s))-f(s, y(s))] d s\right|_{C} \\
& +\mid \exp \left(\int_{t_{0}}^{t} P_{d}(\xi) d \xi\right) \Lambda^{-1}[h(x)-h(y)]-\exp \left(\int_{t_{0}}^{t} P_{d}(\xi) d \xi\right) \Lambda^{-1} \\
& \times \ell\left(\int_{t_{0}}^{t} \exp \left(\int_{s}^{t} P_{d}(\xi) d \xi\right) P_{\bar{d}}(s)[x(s)-y(s)] d s\right. \\
& \left.+\int_{t_{0}}^{t} \exp \left(\int_{s}^{t} P_{d}(\xi) d \xi\right)[f(s, x(s))-f(s, y(s))] d s\right)\left.\right|_{C} \\
\leq & \left(P^{*}+f^{*}\right)|x-y| C \\
& +\Theta_{d}^{*}\left|\Lambda^{-1}\right|\left[H|x-y|_{C}+\bar{\ell}(E)\left(P^{*}+f^{*}\right)|x-y|_{C}\right]
\end{aligned}
$$




$$
=S|x-y|_{C}
$$

where

$$
S=P^{*}+f^{*}+\Theta_{d}^{*}\left|\Lambda^{-1}\right|\left[H+\bar{\ell}(E)\left(P^{*}+f^{*}\right)\right] .
$$

Thus, for $x, y \in C_{0}\left(\mathbb{R}_{+}, \mathbb{R}^{n}\right)$, we get

$$
\|T x-T y\|_{C} \leq\|S\|\|x-y\|_{C}
$$

and since for $S$ is valid $r(S)<1$ we get $\|S\|<1$. Thus we proved that $T$ is a contraction. The existence of the unique solution of the operator equation (2.5) follows from Banach Theorem and by Lemma 1 this solution is also the solution of the problem (1.1), (1.2).

The construction of the solution of problem (1.1), (1.2) follows from Banach Theorem.

Theorem 2. Suppose that all assumptions of Theorem 1 are fulfilled. Then the problem (1.1), (1.2) has the solution $x$ on $\mathbb{R}_{+}$which is a limit of the sequence of the solutions of ancillary system:

$$
\begin{aligned}
& x_{m+1}^{\prime}(t)=P_{d}(t) x_{m+1}(t)+P_{\bar{d}}(t) x_{m}(t)+f\left(t, x_{m}(t)\right), \\
& \ell\left(x_{m+1}\right)=h\left(x_{m}\right),
\end{aligned}
$$

where $m \in \mathbb{N}$ and $x_{1}(t) \in C_{0}\left(\mathbb{R}_{+}, \mathbb{R}^{n}\right)$ is arbitrary.

Proof. It is obvious that the solution to the ancillary system (2.9), (2.10) is equivalent with the solution to the operator equation

$$
x_{m+1}=T x_{m}
$$

where operator $T$ is defined by (2.6), $m \in \mathbb{N}$ and $t^{0} \in \mathbb{R}_{+}$is an arbitrary given point. Let $x_{1}(t)$ be arbitrary. With respect to the contractivity of operator $T$ and to Theorem 1 we shall prove that $x_{2}=T x_{1}, x_{3}=T x_{2}, \ldots, x_{m}=T x_{m-1}, x_{m+1}=T x_{m} \ldots$ is the Cauchy sequence:

$$
\begin{aligned}
\left\|x_{m+1}-x_{m}\right\|_{C} & =\left\|T x_{m}-T x_{m-1}\right\|_{C} \leq\|S\|\left\|x_{m}-x_{m-1}\right\|_{C} \\
& =\|S\|\left\|T x_{m-1}-T x_{m-2}\right\|_{C} \leq\|S\|^{2}\left\|x_{m-1}-x_{m-2}\right\|_{C} \cdots \\
& \leq\|S\|^{m-1}\left\|x_{2}-x_{1}\right\|_{C}
\end{aligned}
$$

where $m \in \mathbb{N}$ is arbitrary. By virtue of this we get for $n>m, n \in \mathbb{N}$

$$
\begin{aligned}
\left\|x_{n}-x_{m}\right\|_{C} & \leq\left\|x_{m+1}-x_{m}\right\|_{C}+\cdots+\left\|x_{n}-x_{n-1}\right\|_{C} \\
& \leq\|S\|^{m-1}\left\|x_{2}-x_{1}\right\|_{C}+\|S\|^{m}\left\|x_{2}-x_{1}\right\|_{C}+\cdots+\|S\|^{n-2}\left\|x_{2}-x_{1}\right\|_{C} \\
& =\left\|x_{2}-x_{1}\right\|_{C}\|S\|^{m-1}\left(1+\|S\|+\cdots+\|S\|^{n-m-1}\right) \\
& \leq\left\|x_{2}-x_{1}\right\|_{C}\|S\|^{m-1}(1+\|S\|+\ldots)=\frac{\|S\|^{m-1}}{1-\|S\|}\left\|x_{2}-x_{1}\right\|_{C}
\end{aligned}
$$


Let $\epsilon>0$ be arbitrary. Since $r(S)<1$ then $\|S\|^{m-1} \rightarrow 0$ for $m \rightarrow \infty$. Thus there exists $n_{0} \in \mathbb{N}$ such that for $m \geq n_{0}$ the following holds:

$$
\|S\|^{m-1}\left\|x_{2}-x_{1}\right\|_{C} \frac{1}{1-\|S\|}<\epsilon
$$

This implies $\left\|x_{n}-x_{m}\right\|_{C}<\epsilon$ for $m, n \geq n_{0}$. Thus we proved that $\left\{x_{m}\right\}$ is a Cauchy sequence. Since $C_{0}$ is a complete space $\left\{x_{m}\right\}$ is convergent. That means $x_{m} \rightarrow x_{0}$, $x_{0} \in C_{0}$. With respect to the Banach Theorem $x_{0}$ is the only one fixed point of the operator $T$ and also the solution of the operator equation (2.11). This solution is also the solution of the problem (2.9), (2.10).

The following Theorem proves that the process of constructing the solution of problem (2.9), (2.10) described above is stable in some sense, analogously to the assertions published by I.Kiguradze in [4].

Theorem 3. Consider a sequence of vectors $\gamma_{m} \in \mathbb{R}^{n}(m=1,2, \ldots)$ and a sequence of vector-valued functions $\Delta_{m} \in L\left(\mathbb{R}_{+}, \mathbb{R}^{n}\right)(m=1,2, \ldots)$. Let $\sum_{m=1}^{\infty} \epsilon_{m}$ be convergent where

$$
\epsilon_{m}=\left(\left\|\gamma_{m}\right\|+\sup \left\{\int_{t_{0}}^{t}\left\|\Delta_{m}(s)\right\| d s: t \in \mathbb{R}\right\}\right)
$$

and assume that all the conditions of Theorem 1 are satisfied. Then the solution $x$ on $\mathbb{R}_{+}$of problem (1.1), (1.2) is the limit of the sequence of solutions of ancillary system:

$$
\begin{aligned}
& x_{m+1}^{\prime}(t)=P_{d}(t) x_{m+1}(t)+P_{\bar{d}}(t) x_{m}(t)+f\left(t, x_{m}(t)\right)+\Delta_{m}(t), \\
& \ell\left(x_{m+1}\right)=h\left(x_{m}\right)+\gamma_{m},
\end{aligned}
$$

where $m \in \mathbb{N}$ and $x_{1}(t) \in C_{0}\left(\mathbb{R}_{+}, \mathbb{R}^{n}\right)$ is arbitrary.

Proof. To prove the theorem we have to show that the operator equation (2.11) enriched with error function doesn't breach the Cauchy property of the sequence occurred in Theorem 2. Therefore we consider the operator equation

$$
x_{m+1}=T x_{m}+\widehat{T}\left(\Delta_{m}, \gamma_{m}\right)
$$

where operator $T$ is defined by (2.6) and

$$
\begin{aligned}
\widehat{T}\left(\Delta_{m}, \gamma_{m}\right) & =\int_{t_{0}}^{t} \exp \left(\int_{s}^{t} P_{d}(\xi) d \xi\right) \Delta_{m}(s) d s \\
& +\exp \left(\int_{t_{0}}^{t} P_{d}(\xi) d \xi\right) \Lambda^{-1}\left[\gamma_{m}-\ell\left(\int_{t_{0}} \exp \left(\int_{s} P_{d}(\xi) d \xi\right) \Delta_{m}(s) d s\right)\right]
\end{aligned}
$$

where $m \in \mathbb{N}$ and $t^{0} \in \mathbb{R}_{+}$is an arbitrary given point. Now, to prove the stability of the solution we show that also the sequence $x_{2}=T x_{1}+\widehat{T}\left(\Delta_{1}, \gamma_{1}\right), \ldots, x_{m}=$ $T x_{m-1}+\widehat{T}\left(\Delta_{m-1}, \gamma_{m-1}\right), x_{m+1}=T x_{m}+\widehat{T}\left(\Delta_{m}, \gamma_{m}\right) \ldots$ is a Cauchy sequence, 
with respect to Theorem 1, 2 and to the contractivity of operator $(T+\widehat{T})$, analogously to the proof of Theorem 2. In addition to (2.8) we need to make the following estimation:

$$
\begin{aligned}
\left\|\widehat{T}\left(\Delta_{m}, \gamma_{m}\right)-\widehat{T}\left(\Delta_{m-1}, \gamma_{m-1}\right)\right\| \\
\leq\left\|\int_{t_{0}}^{t} \exp \left(\int_{s}^{t} P_{d}(\xi) d \xi\right)\left[\Delta_{m}(s) d s-\Delta_{m-1}(s)\right] d s\right\| \\
\quad+\left\|\exp \left(\int_{t_{0}}^{t} P_{d}(\xi) d \xi\right) \Lambda^{-1}\left[\gamma_{m}-\gamma_{m-1}\right]\right\| \\
\quad+\left\|\exp \left(\int_{t_{0}}^{t} P_{d}(\xi) d \xi\right) \Lambda^{-1} \ell\left(\int_{t_{0}} \exp \left(\int_{s} P_{d}(\xi) d \xi\right)\left[\Delta_{m}(s)-\Delta_{m-1}\right] d s\right)\right\| \\
\leq\left\|\Theta_{d}^{*}\left|\Lambda^{-1}\right|\left[\gamma_{m}-\gamma_{m-1}\right]\right\| \\
\quad+\left\|\Theta_{d}^{*}\left(1+\left|\Lambda^{-1}\right| \bar{\ell}(E) \Theta_{d}^{*}\right) \int_{t_{0}}^{t}\left[\Delta_{m}(s) d s-\Delta_{m-1}(s)\right] d s\right\| \\
\leq\left\|c^{*}\right\|\left\|\gamma_{m}-\gamma_{m-1}\right\|+\left\|S_{\Delta}\right\| \int_{t_{0}}^{t}\left\|\Delta_{m}(s) d s-\Delta_{m-1}(s)\right\| d s
\end{aligned}
$$

where

$$
c^{*}=\Theta_{d}^{*}\left|\Lambda^{-1}\right|, \quad S_{\Delta}=\Theta_{d}^{*}\left(1+\left|\Lambda^{-1}\right| \bar{\ell}(E) \Theta_{d}^{*}\right) .
$$

Then

$$
\begin{aligned}
\left\|x_{m+1}-x_{m}\right\|_{C}= & \left\|T x_{m}+\widehat{T}\left(\Delta_{m}, \gamma_{m}\right)-\left(T x_{m-1}+\widehat{T}\left(\Delta_{m-1}, \gamma_{m-1}\right)\right)\right\|_{C} \\
\leq & \left\|T_{x_{m}}-T x_{m-1}\right\|_{C}+\left\|\widehat{T}\left(\Delta_{m}, \gamma_{m}\right)-\widehat{T}\left(\Delta_{m-1}, \gamma_{m-1}\right)\right\| \\
\leq & \|S\|\left\|x_{m}-x_{m-1}\right\|+\left\|c^{*}\right\|\left\|\gamma_{m}-\gamma_{m-1}\right\| \\
& +\left\|S_{\Delta}\right\| \int_{t_{0}}^{t}\left\|\Delta_{m}(s) d s-\Delta_{m-1}(s)\right\| d s \\
\leq & \|S\|\left\|T_{x_{m-1}}-T x_{m-2}\right\|_{C}+\left\|\widehat{T}\left(\Delta_{m-1}, \gamma_{m-1}\right)-\widehat{T}\left(\Delta_{m-2}, \gamma_{m-2}\right)\right\| \\
& +\left\|c^{*}\right\|\left\|\gamma_{m}-\gamma_{m-1}\right\|+\left\|S_{\Delta}\right\| \int_{t_{0}}^{t}\left\|\Delta_{m}(s) d s-\Delta_{m-1}(s)\right\| d s \leq \ldots \\
\leq & \|S\|^{m-1}\left\|x_{2}-x_{1}\right\| \\
& +\left\|c^{*}\right\| \sum_{i=2}^{m}\left\|\gamma_{i}-\gamma_{i-1}\right\|+\left\|S_{\Delta}\right\| \sum_{i=2}^{m} \int_{t_{0}}^{t}\left\|\Delta_{i}(s)-\Delta_{i-1}(s)\right\| d s
\end{aligned}
$$

where $m \in \mathbb{N}$ is arbitrary. For $n>m, n \in \mathbb{N}$ we get

$$
\left\|x_{n}-x_{m}\right\|_{C}=\frac{\|S\|^{m-1}}{1-\|S\|}\left\|x_{2}-x_{1}\right\|_{C}+\left\|c^{*}\right\| \sum_{i=2}^{m}\left\|\gamma_{i}-\gamma_{i-1}\right\|
$$




$$
\begin{aligned}
& +\left\|S_{\Delta}\right\| \sum_{i=2}^{m} \int_{t_{0}}^{t}\left\|\Delta_{i}(s)-\Delta_{i-1}(s)\right\| d s \\
& +\left\|c^{*}\right\| \sum_{i=2}^{m+1}\left\|\gamma_{i}-\gamma_{i-1}\right\|+\left\|S_{\Delta}\right\| \sum_{i=2}^{m+1} \int_{t_{0}}^{t}\left\|\Delta_{i}(s)-\Delta_{i-1}(s)\right\| d s+ \\
& \vdots \\
& +\left\|c^{*}\right\| \sum_{i=2}^{n-1}\left\|\gamma_{i}-\gamma_{i-1}\right\|+\left\|S_{\Delta}\right\| \sum_{i=2}^{n-1} \int_{t_{0}}^{t}\left\|\Delta_{i}(s)-\Delta_{i-1}(s)\right\| d s \\
& +\left\|c^{*}\right\| \sum_{i=2}^{n}\left\|\gamma_{i}-\gamma_{i-1}\right\|+\left\|S_{\Delta}\right\| \sum_{i=2}^{n} \int_{t_{0}}^{t}\left\|\Delta_{i}(s)-\Delta_{i-1}(s)\right\| d s \\
& \leq \frac{\|S\|^{m-1}}{1-\|S\|}\left\|x_{2}-x_{1}\right\| C \\
& +\sum_{j=m}^{n}\left(\left\|c^{*}\right\|\left\|\gamma_{j}\right\|+\left\|S_{\Delta}\right\| \sup \left\{\int_{t_{0}}^{t}\left\|\Delta_{j}(s)\right\| d s: t \in \mathbb{R}\right\}\right) .
\end{aligned}
$$

Convergency of $\sum_{j=1}^{n} \epsilon_{j}$ implies the convergency of the series of both addends of $\epsilon_{m}$ and their linear combinations as well. Then by virtue of Theorem 2 the sequence $\left\{x_{m}\right\}=\left\{T x_{m}+\widehat{T}\left(\Delta_{m}, \gamma_{m}\right)\right\}$ is Cauchy and $x_{m}=T x_{m}+\widehat{T}\left(\Delta_{m}, \gamma_{m}\right) \rightarrow x_{0} \in C_{0}$ which is the solution of the problem (2.12), (2.13).

Remark 1. If $\gamma_{m}=0$ and $\Delta_{m} \equiv 0$ then from Theorem 3 we get immediately Theorem 2. Thus Theorem 2 could be perceived as the corollary of Theorem 3. For clearer view on calculations in proofs of both theorems it is convenient to formulate first Theorem 2 separately and then Theorem 3 including the error function.

All consequences for linear boundary value problems published in [3] and analogous statements for solutions of linear boundary value problems for nonlinear systems of ordinary differential equations follow directly from Theorem 1 and Theorem 2 .

The following example illustrates the case of nonlinear boundary value problem for nonlinear system of ordinary differential equations:

$$
\begin{aligned}
& x_{i}^{\prime}(t)=-x_{i}(t)+g_{i}(t) \sin x_{i}(t)+q_{i}(t), \quad(i=1, \ldots, n), \\
& x_{i}(0)=c_{i} \cos x_{i}(1)+c_{0 i}, \quad(i=1, \ldots, n),
\end{aligned}
$$

where $g_{i}, q_{i} \in L_{l o c}\left(\mathbb{R}_{+}, \mathbb{R}\right), c_{i}, c_{0 i} \in \mathbb{R}(i=1, \ldots, n)$.

Then we have

$$
\begin{aligned}
& P(t) \equiv-E \equiv P_{d}(t), \quad P_{\bar{d}}(t) \equiv 0, \\
& f(t, x)=\left(g_{i}(t) \sin x_{i}(t)+q_{i}(t)\right)_{i=1}^{n},
\end{aligned}
$$




$$
l(x)=\left(x_{i}(0)\right)_{i=1}^{n}, \quad h(x)=\left(c_{i} \cos x_{i}(1)+c_{0 i}\right)_{i=1}^{n}
$$

and then

$$
|f(t, x)| \leq\left(\left|g_{i}(t)\right|+\left|q_{i}(t)\right|\right)_{i=1}^{n}, \quad|h(x)| \leq\left(\left|c_{i}\right|+\left|c_{0 i}\right|\right)_{i=1}^{n}, \quad \bar{L}(t) \equiv 0, \quad \bar{h}=0 .
$$

Because $P_{d}^{*}=0, P^{*}=0, \bar{\ell}(E)=E$ then it follows that condition (2.1) is fulfilled on assumption

$$
\|g\|_{L}+\|q\|_{L}<+\infty
$$

Then $S=0$ which means that the differential equation has a unique solution.

\section{REFERENCES}

[1] R. P. Agarwal and D. O'Regan, Infinite interval problems for differential, difference and integral equations. Kluwer Academic Publishers, Dordrecht, 2001. doi: 10.1007/978-94-010-0718-4.

[2] V. M. Evtukhov and L. I. Kusick, "Asymptotic behaviour of solutions of some systems of quasilinear differential equations," Math. Notes (Miskolc), vol. 4, no. 1, pp. 3-24, 2003.

[3] J. Jandová and B. Půža, "On the solvability of a linear boundary value problems for system of ordinary differential equations on $\mathbb{R}_{+}$," in Colloquium on Differential and Difference Equations$C D D E$ 2006, ser. Folia Fac. Sci. Natur. Univ. Masaryk. Brun. Math. Masaryk Univ., Brno, 2007, vol. 16 , pp. $75-83$.

[4] I. Kiguradze, Nachalnaya i kraevye zadachi dlya sistem obyknovennykh differentsialnykh uravnenii. Tom I. "Metsniereba", Tbilisi, 1997, lineinaya teoriya. [Linear theory].

[5] I. Kiguradze, "On some boundary value problems with conditions at infinity for nonlinear differential systems," Bull. Georgian Natl. Acad. Sci., vol. 175, no. 1, pp. 27-33, 2007.

[6] I. Kiguradze and B. Půža, Boundary value problems for systems of linear functional differential equations, ser. Folia Facultatis Scientiarium Naturalium Universitatis Masarykianae Brunensis. Mathematica. Masaryk University, Brno, 2003, vol. 12.

[7] I. Kiguradze and Z. Sokhadze, "A priori estimates of solutions of systems of functional differential inequalities and some of their applications," Mem. Differential Equations Math. Phys., vol. 41, pp. 43-67, 2007.

[8] N. A. Perestyuk and V. Y. Slusarchuk, "Oscillation of nonlinear differential-integral equation in a Banach space with respect to its subspace," Math. Notes (Miskolc), vol. 4, no. 1, pp. 53-64, 2003.

Author's address

Jitka Vacková

Department of Mathematics and Statistics, Masaryk University Brno, Czech Republic

E-mail address: jitka@finnsub.com 